\title{
FISH BYCATCH OF THE LAULAO CATFISH Brachyplatystoma vaillantii (VALENCIENNES, 1840) TRAWL FISHERY IN THE AMAZON ESTUARY
}

\author{
Erica Antunes Jimenez, ${ }^{1, *}$, Mutsuo Asano Filho ${ }^{2}$ and Flávia Lucena Frédou ${ }^{3}$ \\ ${ }^{1}$ Universidade Federal do Pará - Programa de Pós-Graduação em Biologia Ambiental \\ (Av. Leandro Ribeiro, s/n, Aldeia, 68600-000 Bragança, PA, Brasil) \\ ${ }^{2}$ Ministério da Pesca e Aqüicultura \\ (Setor Bancário Sul, Quadra 02, Lote 10, 5 andar, Ed. Carlton Tower, 70070-120 Brasília, DF, Brasil) \\ ${ }^{3}$ Universidade Federal Rural de Pernambuco \\ (Rua Dom Manoel de Medeiros, s/n, Dois Irmãos, 52171-900 Recife, PE, Brasil) \\ *Correspondence author: ericaajimenez@gmail.com
}

\begin{abstract}
A B S TRACT
This study aimed to analyse the composition and seasonal variation in the fish bycatch of the Brachyplatystoma vaillantii trawl fishery in the Amazon Estuary in 2009 by monitoring the trips of 48 vessels. The bycatch represented $29 \%$ of the catches, totalling 22,228 specimens and 52 taxa, distributed in 22 families (the principal families were Ariidae, Pimelodidae, and Sciaenidae). Brachyplatystoma rousseauxii, Plagioscion squamosissimus, and Sciades herzbergii together contributed $69 \%$ of the bycatch and were considered consistent bycatch species. Although a higher proportion of bycatch was captured during the rainy season, the seasonal difference was not significant. A multidimensional scaling (MDS) ordination analysis and an analysis of similarity (ANOSIM) indicated that the species composition of the bycatch was similar across the seasons. However, larger numbers of $B$. rousseauxii and $P$. squamosissimus were captured during the rainy season, whereas $S$. herzbergii predominated during the dry season. The marine migrants and estuarine species guilds showed the greatest richness, whereas freshwater migrants were the most numerous. Among the feeding guilds, the zoobenthivores were the most diverse, whereas the piscivores were the most abundant. The results indicate that fishing pressure primarily affects small$(20-30 \mathrm{~cm})$ and medium-sized $(30-50 \mathrm{~cm})$ individuals, although the catch of $P$. squamosissimus was composed primarily of adults. However, the catches of both $B$. rousseauxii and B. vaillantii were composed primarily of juveniles.
\end{abstract}

\section{RESUMO}

Este estudo objetivou analisar a composição e variação sazonal da ictiofauna acompanhante da pesca de arrasto de Brachyplatystoma vaillantii no estuário amazônico, em 2009, através do monitoramento de viagens de 48 embarcações. A ictiofauna acompanhante representou $29 \%$ das capturas, totalizando 22.228 indivíduos e 52 táxons pertencentes a 22 famílias, sendo estas as principais: Ariidae, Pimelodidae e Sciaenidae. Brachyplatystoma rousseauxii, Plagioscion squamosissimus e Sciades herzbergii representaram juntas $69 \%$ da captura e foram consideradas constantes. Embora o maior percentual de captura tenha ocorrido no período chuvoso, as diferenças sazonais não foram significativas. Análises de ordenação (MDS) e de similaridade (ANOSIM) mostraram que a composição da captura entre os períodos é semelhante; entretanto, maiores capturas de $B$. rousseauxii e $P$. squamosissimus foram observadas no período chuvoso, enquanto $S$. herzbergii foi dominante no período seco. As guildas migrantes marinhas e espécies estuarinas apresentaram maior riqueza, enquanto as migrantes dulcícolas predominaram em percentual de indivíduos. Considerando as guildas alimentares, as zoobentívoras apresentaram a maior riqueza, enquanto as piscívoras predominaram em abundância relativa. Os resultados indicam que a pressão pesqueira atua sensivelmente sobre os indivíduos de pequeno $(20-30 \mathrm{~cm})$ e médio $(30-50 \mathrm{~cm})$ porte, embora a captura de $P$. squamosissimus seja composta predominantemente por adultos. A captura de $B$. rousseauxii e $B$. vaillantii, entretanto, foi composta predominantemente por juvenis.

Descriptors: Industrial fishing, Diversity, Juveniles, Catfish.

Descritores: Pesca industrial, Diversidade, Juvenis, Bagre.

\section{INTRODUCTION}

In the early 1990s, when fisheries throughout the world began to approach the theoretical limits of fish production and competition for limited resources grew alarmingly, the need for the more rational exploitation of fishery resources became increasingly apparent. The capture of bycatch has a number of negative effects from an ecological 
perspective. Bycatch also causes economic and biological losses (ALVERSON; HUGHES, 1996; HALL; MAINPRIZE, 2005). One of the major problems associated with bycatch, as the result of nonselective fishing practices, is that bycatch may include juveniles of commercial and non-commercial species or individuals of threatened, protected or endangered species (ALVERSON et al., 1994; KENELLY, 1995; LEWISON et al., 2004). Another negative aspect is the discarding of fish (SAILA, 1983; ALVERSON et al., 1994; STOBUTZKI et al., 2003), which constitutes a serious problem for managers because it represents an unquantified mortality variable in fisheries (ALVERSON; HUGHES, 1996). Consequently, populations that are subject to bycatch can decline over short timescales, often without previous detection (CASEY; MYERS, 1998).

From an ecological perspective, the capture of bycatch can produce several negative effects on the ecosystem, including loss of biodiversity and changes in habitat and densities of predators and prey (LEWISON et al., 2004; STOBUTSKI et al., 2003; CLUCAS, 1997; MURRAY et al., 1992).

An estimated seven million tons of bycatch are discarded by fisheries worldwide each year (KELLEHER, 2005). Off the coast of northern Brazil, approximately 30 thousand tons of bycatch are discarded annually by marine and estuarine fisheries, in particular by trawlers (ISAAC, 1998). In the Amazon Estuary, industrial trawling is one of the most important fishery activities in terms of both the volume of the catch landed and the income derived from this activity (IBAMA, 2007). One of the principal targets of the industrial trawler fleets of northern Brazil is the laulao, Brachyplatystoma vaillantii (Valenciennes, 1840), a freshwater catfish of the Pimelodidae family, which is widely distributed in the river basins of northern South America (MEES, 1974).

The fishery for large migratory catfishes, such as the laulao catfish, is conducted in almost the entire Amazon basin. Brazilian, Colombian and Peruvian fishermen jointly exploit an area that extends from Tefé, in the Middle Solimões River, to Iquitos, in Peru. The main landing ports are located in Pará State and in Letícia, Colombia (BARTHEM; GOULDING, 1997; FABRÉ; ALONSO, 1998; FABRÉ et al., 2000; PARENTE et al., 2005). Nearly ten thousand tons of fish are landed per year in Leticia.

In Brazil, the peak of laulao captures occurred in the $1970 \mathrm{~s}$, when approximately 29 thousand tons were landed. This period also corresponded to the peak of tax incentives and investments in fishing equipment. During the ' $80 \mathrm{~s}$, however, the relative laulao catfish stock showed signs of depletion (BARTHEM; GOULDING, 1997), and the stock was classified as overfished (BARTHEM;
PETRERE, 1995). Currently, the stock shows signs of recovery. Between 2007 and 2010, the fishery landed an average of approximately 24,400 tons per year (MPA, 2012). During this period, approximately 2,300 tons of this species were exported, generating an income of more than US\$ 2 million (MDIC/ALICEWEB, 2007-2010). Isaac (1998) estimated that approximately $12 \%$ of the $B$. vaillantii catch is discarded as bycatch.

The objective of the present study was to contribute to the discussion of the impact and potential management of bottom trawl fisheries. Specifically, the study analysed the composition and seasonal variation of the fish fauna captured as bycatch during industrial trawling for laulao catfish in the Amazon Estuary.

\section{Material And Methods}

Study Area

The Amazon Estuary has megathermic weather, with a relative humidity above $80 \%$ and a mean air temperature of $25^{\circ} \mathrm{C}$. The region has two distinct seasons: rainy (January to June) and dry (July to December) (MARTORANO et al., 1993). The average annual rainfall is 2,214 mm (MORAES et al., 2005).

\section{Data Collection}

Approximately $20 \%$ of the trips of the Amazonian laulao trawling fleet (48 vessels) were monitored between January and December, 2009. This fleet conducts pair trawling in the Amazon Estuary between latitudes $00^{\circ} 03.7^{\prime} \mathrm{N}$ and $01^{\circ} 40.7^{\prime} \mathrm{N}$ and longitudes $48^{\circ} 5.7^{\prime} \mathrm{W}$ and $49^{\circ} 54.4^{\prime} \mathrm{W}$ (Fig. 1). Overall, 575 trawl hauls were examined. In each case, five baskets of fish were sampled randomly. Prior to landing, the specimens were identified, and the total (TL), fork (FL), and standard (SL) lengths were measured.

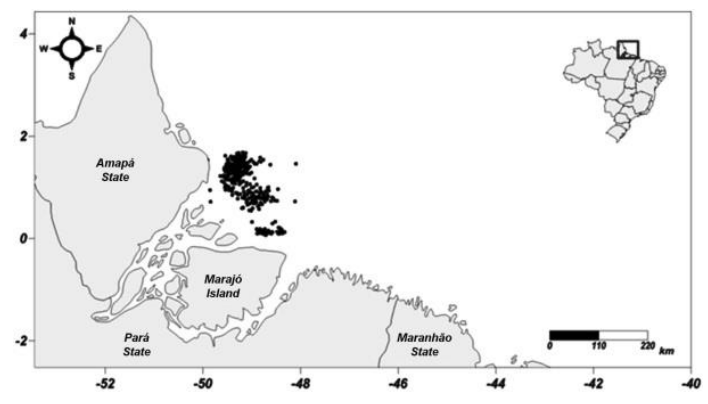

Fig. 1. Operational area of the industrial trawler fleet that targets B. vaillantii off the northern coast of Brazil in 2009. 


\section{Data Analysis}

\section{Catch Composition and Frequency of Occurrence}

The composition of the catches was evaluated in terms of their species richness, relative frequency, and frequency of occurrence $(\% \mathrm{~F})$. These parameters were calculated for the entire study period and separately for the rainy and dry seasons, which were defined as in Martorano et al. (1993). The seasonal variation in the proportion of bycatch was evaluated using a chi-square test at a 5\% significance level. Between-season differences in species richness were tested with a one-way ANOVA. Shapiro-Wilk and Cochran tests were used to test the normality and homoscedasticity of the data, respectively. If necessary, the data were $\log (x+1)$ transformed.

The frequency of occurrence of the different species was classified according to the scheme of Dajoz (1973), in which a frequency of occurrence $(\% \mathrm{~F})$ value of $50 \%$ or above is considered to be constant, 25-50\% accessory, and less than 25\% occasional. A multidimensional scaling (MDS) ordination analysis was used to evaluate the similarities in the structure of the fish assemblage between seasons. An analysis of similarity (ANOSIM) was used to test whether the groups found by MDS were statistically different (CLARKE, 1993).

\section{Ecological Guilds}

The available data on the ecology of the identified fish species in transitional environments was used to classify the species according to two functional features (based on Elliot et al., 2007): (1) the way the fish uses the estuary over the course of its life cycle, i.e., the Estuarine Use Functional Group, or EUFG, and (2) its feeding preferences and strategies, known as the Feeding Mode Functional Group, or FMFG.

All species were allocated to one of the environmental guilds (EUFGs) defined by Elliott et al. (2007): Marine Stragglers (MS), Marine Migrants (MM), Estuarine Species (ES), Freshwater Migrants (FM), and Freshwater Stragglers (FS). These guilds were defined primarily in terms of the type, frequency, and period of use of the estuarine environment and the abundance of the species in the estuary.

The feeding guilds (FMFGs) were defined according to the available literature (KRUMME et al., 2004; ELLIOTT et al., 2007). The species were classified as zooplanktivore (ZP), detritivore (DV), herbivore (HV), piscivore (PV), zoobenthivore (ZB), omnivore/opportunist (OV/OP), and piscivore/zoobenthivore (PV/ZB). The latter two categories were included based on data collected in the present study. The FMFGs were identified by combining the data available on the predominant diet and feeding site. If few data were available for a species, trophic preferences were inferred from the information gathered by the Fishbase project (FROESE; PAULY, 2010). The guilds were based on the percentage of both species and number of individuals, following Selleslagh et al. (2009).

\section{Body Length and Catch Probability}

The cumulative frequency of the body lengths of the target and principal bycatch species was adjusted to a logistic model to estimate the length at which the catch probability is $50 \%\left(\mathrm{P}_{50}\right)$. The $\mathrm{P}_{50}$ values were compared with those for first sexual maturity $\left(\mathrm{L}_{50}\right)$ available in Vásquez et al. (2009) for $B$. vaillantii, Fabré et al. (2000) for Brachyplatystoma rousseauxii, and Barbosa et al. (2012) for Plagioscion squamosissimus.

\section{RESULTS}

\section{Catch Composition}

A total of 75,714 specimens were collected during the study period. The bycatch represented $29 \%$ of the catches, totalling 22,228 specimens and 52 taxa, of which 41 were identified to species level, representing 22 families (Table 1). Species richness was similar between seasons, with 47 species observed in the rainy season and 42 in the dry season (ANOVA, $\mathrm{p}>0.05)$. The Sciaenidae, Pimelodidae, and Ariidae families together represented $44 \%$ of the total number of species and $91 \%$ of the specimens collected (Table 1).

Overall, $47 \%$ of the species captured were classified as occasional (F $<25 \%$ ), whereas Pellona flavipinnis and Lithodoras dorsalis were classified as accessory. Only B. rousseauxii, $P$. squamosissimus, and Sciades herzbergii were considered to be consistent and essential members of the bycatch from industrial laulao trawling. Individuals of these three species represented $39.9 \%, 17.4 \%$, and $11.2 \%$, respectively, of the total bycatch (Table 1), together constituting approximately $69 \%$ of the sample.

Taxa recorded exclusively in only one of the two seasons were relatively rare. Five taxa (Cathorops spixii, Genyatremus luteus, Mugil sp., Plagioscion auratus, and Stellifer spp.) were captured exclusively in the dry season and ten in the rainy season (Colomesus psittacus, Oligoplites sp., Scomberomorus brasiliensis, Acanthocybium solandri, Hypostomus punctatus, Eigenmannia virescens, Polydactylus virginicus, Trinectes paulistanus, Dasyatis geijskesi, and jururema). A total of 37 taxa occurred throughout the study period. 
Although the bycatch represented a higher percentage during the rainy season than during the dry season, the difference was not significant (chi-square, $\mathrm{P}>$ 0.05). Among the principal species, the highest relative frequency of $S$. herzbergii occurred during the dry season $(14.4 \%)$, whereas $B$. rousseauxii $(46.1 \%)$ and $P$. squamosissimus $(23 \%)$ were captured in higher numbers during the rainy season (Table 1).

A MDS ordination analysis found no evidence of distinct species groups between seasons (Fig. 2). The $R$ value for the ANOSIM was also relatively low $(R=0.113)$. This value indicates that between- and within-group similarity is high.

Table 1. Absolute and relative frequency (total and by seasons), minimum (Min) and maximum (Max) lengths (in centimetres $\mathrm{cm})$ and ecological guilds of species captured as bycatch of laulao trawling in the Amazon estuary. Total Length (*); Fork Length (**); Feeding Mode Functional Group (FMFG); Zooplanktivore (ZP); Detritivore (DV); Herbivore (HV); Piscivore $(\mathrm{PV})$; Zoobenthivore (ZB); Piscivore/Zoobenthivore (PV/ZB); Estuarine Use Functional Group (EUFG); Marine Stragglers (MS); Marine Migrants (MM); Estuarine Species (ES); Freshwater Migrants (FM); and Freshwater Stragglers (FS).

\begin{tabular}{|c|c|c|c|c|c|c|c|c|c|c|c|c|}
\hline \multirow[t]{2}{*}{ Family } & \multirow[t]{2}{*}{ Scientific name } & \multicolumn{3}{|c|}{ Absolute Frequency } & \multicolumn{3}{|c|}{ Relative Frequency } & \multicolumn{2}{|c|}{$\begin{array}{l}\text { Length Ranges } \\
(\mathrm{cm})\end{array}$} & \multicolumn{3}{|c|}{ Guilds } \\
\hline & & Total & Rainy & Dry & Total & Rainy & Dry & Min & Max & $\begin{array}{l}\text { FM } \\
\text { FG }\end{array}$ & EUFG & Source \\
\hline \multicolumn{13}{|c|}{ Achiridae } \\
\hline & Achirus sp. & 10 & 8 & 2 & 0.04 & 0.07 & 0.02 & $17.0^{*}$ & $30.0^{*}$ & - & - & \\
\hline & $\begin{array}{l}\text { Apionichthys dumerili } \\
\text { Kaup, } 1858\end{array}$ & 14 & 7 & 7 & 0.06 & 0.06 & 0.06 & $16.0^{*}$ & $42.0^{*}$ & - & FM & $\begin{array}{l}\text { Froese \& Pauly } \\
\text { (2010) }\end{array}$ \\
\hline & $\begin{array}{l}\text { Trinectes paulistanus } \\
\text { (Miranda Ribeiro, } \\
\text { 1915) }\end{array}$ & 8 & 8 & - & 0.04 & 0.07 & - & $20.0^{*}$ & $40.0^{*}$ & $\mathrm{ZB}$ & MM & $\begin{array}{l}\text { Froese \& Pauly } \\
\text { (2010) }\end{array}$ \\
\hline \multicolumn{13}{|c|}{ Ageneiosidae } \\
\hline & $\begin{array}{l}\text { Ageneiosus inermis } \\
\text { (Linnaeus, 1766) }\end{array}$ & 90 & 56 & 34 & 0.40 & 0.50 & 0.31 & $17.0^{* * *}$ & $53.0^{* * *}$ & $\begin{array}{l}\mathrm{PV} / \\
\mathrm{ZB}\end{array}$ & FS & $\begin{array}{l}\text { Froese \& Pauly } \\
\text { (2010); }\end{array}$ \\
\hline \multicolumn{13}{|l|}{ Ariidae } \\
\hline & $\begin{array}{l}\text { Amphiarius rugispinis } \\
\text { (Valenciennes 1840) }\end{array}$ & 285 & 31 & 254 & 1.28 & 0.28 & 2.29 & $15.0^{* * *}$ & $47.0^{* *}$ & $\mathrm{ZB}$ & ES & $\begin{array}{l}\text { Mendes \& } \\
\text { Barthem } \\
\text { (2010); Espírito } \\
\text { Santo et al. } \\
\text { (2005) }\end{array}$ \\
\hline & $\begin{array}{l}\text { Aspistor quadriscutis } \\
\text { (Valenciennes, 1840) }\end{array}$ & 1122 & 286 & 836 & 5.05 & 2.56 & 7.55 & $14.0^{* *}$ & $63.0^{* *}$ & $\mathrm{ZB}$ & ES & $\begin{array}{l}\text { Barros et al. } \\
\text { (2011); Froese } \\
\text { \& Pauly (2010); } \\
\text { Mendes \& } \\
\text { Barthem (2010) }\end{array}$ \\
\hline & $\begin{array}{l}\text { Bagre bagre } \\
\text { (Linnaeus, 1766) }\end{array}$ & 538 & 188 & 350 & 2.42 & 1.69 & 3.16 & $18.0^{* * *}$ & $63.2^{* * *}$ & $\begin{array}{l}\mathrm{PV} / \\
\mathrm{ZB}\end{array}$ & MM & $\begin{array}{l}\text { Froese \& Pauly } \\
\text { (2010); Léopold } \\
\text { (2004) }\end{array}$ \\
\hline & $\begin{array}{l}\text { Cathorops spixii } \\
\text { (Agassiz, 1829) }\end{array}$ & 1 & - & 1 & 0.00 & - & 0.01 & $27.0^{* *}$ & $27.0^{* * *}$ & $\mathrm{ZB}$ & ES & $\begin{array}{l}\text { Barletta \& } \\
\text { Blaber (2007) }\end{array}$ \\
\hline & $\begin{array}{l}\text { Notarius grandicassis } \\
\text { (Valenciennes, 1840) }\end{array}$ & 370 & 210 & 160 & 1.66 & 1.88 & 1.44 & $16.0^{* *}$ & $72.0^{* * *}$ & $\mathrm{ZB}$ & ES & $\begin{array}{l}\text { Froese \& Pauly } \\
\text { (2010); Léopold } \\
\text { (2004); } \\
\text { Krumme et al. } \\
(2004)\end{array}$ \\
\hline & $\begin{array}{l}\text { Sciades herzbergii } \\
\text { (Bloch, 1794) }\end{array}$ & 2498 & 898 & 1600 & 11.24 & 8.05 & 14.45 & $10.0^{* * *}$ & $130.0^{* * *}$ & $\mathrm{ZB}$ & ES & $\begin{array}{l}\text { Krumme et al. } \\
\text { (2008); } \\
\text { Giarrizzo \& } \\
\text { Saint-Paul } \\
\text { (2008); Barletta } \\
\text { \& Blaber } \\
\text { (2007); } \\
\text { Krumme et al. } \\
\text { (2004) }\end{array}$ \\
\hline & $\begin{array}{l}\text { Sciades parkeri } \\
\text { (Traill, 1832) }\end{array}$ & 425 & 140 & 285 & 1.91 & 1.26 & 2.57 & $10.0^{* *}$ & $140.0^{* * *}$ & $\begin{array}{l}\mathrm{PV} / \\
\mathrm{ZB}\end{array}$ & ES & $\begin{array}{l}\text { Froese \& Pauly } \\
\text { (2010) }\end{array}$ \\
\hline & $\begin{array}{l}\text { Sciades proops } \\
\text { (Valenciennes, 1840) }\end{array}$ & 84 & 16 & 68 & 0.38 & 0.14 & 0.61 & $17.0^{* * *}$ & $76.0^{* * *}$ & PV & ES & $\begin{array}{l}\text { Barros et al. } \\
\text { (2011); Froese } \\
\text { \& Pauly (2010) }\end{array}$ \\
\hline \multicolumn{13}{|c|}{ Aspredinidae } \\
\hline & $\begin{array}{l}\text { Aspredo aspredo } \\
\text { (Linnaeus, 1758) }\end{array}$ & 264 & 144 & 120 & 1.19 & 1.29 & 1.08 & $15.0^{*}$ & $50.0^{*}$ & DV & ES & $\begin{array}{l}\text { Froese \& Pauly } \\
\text { (2010); Espírito } \\
\text { Santo et al. } \\
\text { (2005); Léopold } \\
\text { (2004); } \\
\text { Camargo \& } \\
\text { Isaac (1998) }\end{array}$ \\
\hline \multicolumn{13}{|c|}{ Auchenipteridae } \\
\hline & $\begin{array}{l}\text { Pseudauchenipterus } \\
\text { nodosus (Bloch, 1794) }\end{array}$ & 38 & 8 & 30 & 0.17 & 0.07 & 0.27 & $15.0^{* *}$ & $53.0^{* * *}$ & $\mathrm{ZB}$ & FS & $\begin{array}{l}\text { Barros et al. } \\
(2011) ; \\
\text { Krumme et al. } \\
(2004)\end{array}$ \\
\hline
\end{tabular}


Table 1. Cont

\begin{tabular}{|c|c|c|c|c|c|c|c|c|c|c|c|c|}
\hline \multirow[t]{2}{*}{ Family } & \multirow[t]{2}{*}{ Scientific name } & \multicolumn{3}{|c|}{ Absolute Frequency } & \multicolumn{3}{|c|}{ Relative Frequency } & \multicolumn{2}{|c|}{$\begin{array}{l}\text { Length Ranges } \\
(\mathrm{cm})\end{array}$} & \multicolumn{3}{|c|}{ Guilds } \\
\hline & & Total & Rainy & Dry & Total & Rainy & Dry & Min & Max & $\begin{array}{l}\text { FM } \\
\text { FG }\end{array}$ & EUFG & Source \\
\hline \multicolumn{13}{|c|}{ Batrachoididae } \\
\hline & $\begin{array}{l}\text { Batrachoides } \\
\text { surinamensis (Bloch \& } \\
\text { Schneider, 1801) }\end{array}$ & 22 & 10 & 12 & 0.10 & 0.09 & 0.11 & $31.0^{*}$ & $62.0^{*}$ & $\begin{array}{l}\mathrm{PV} / \\
\mathrm{ZB}\end{array}$ & ES & $\begin{array}{l}\text { Barletta \& } \\
\text { Blaber (2007); } \\
\text { Krumme et al. } \\
(2004)\end{array}$ \\
\hline \multicolumn{13}{|c|}{ Carangidae } \\
\hline & Oligoplites sp. & 1 & 1 & - & 0.00 & 0.01 & - & $33.0^{* * *}$ & $33.0^{* * *}$ & - & - & \\
\hline \multicolumn{13}{|c|}{ Centropomidae } \\
\hline & Centropomus sp. & 6 & 1 & 5 & 0.03 & 0.01 & 0.05 & $30.0^{* * *}$ & $68.0^{* *}$ & - & - & \\
\hline \multicolumn{13}{|c|}{ Dasyatidae } \\
\hline & $\begin{array}{l}\text { Dasyatis geijskesi } \\
\text { Boeseman, } 1948\end{array}$ & 22 & 22 & - & 0.10 & 0.20 & - & $30.0^{*}$ & $97.0^{*}$ & $\mathrm{ZB}$ & MS & $\begin{array}{l}\text { Froese \& Pauly } \\
\text { (2010); Espírito } \\
\text { Santo et al. } \\
\text { (2005); Léopold } \\
\text { (2004) }\end{array}$ \\
\hline \multicolumn{13}{|c|}{ Doradidae } \\
\hline & $\begin{array}{l}\text { Lithodoras dorsalis } \\
\text { (Valenciennes, 1840) }\end{array}$ & 548 & 324 & 224 & 2.47 & 2.91 & 2.02 & $12.0^{* *}$ & $60.2 * *$ & HV & FS & $\begin{array}{l}\text { Froese \& Pauly } \\
\text { (2010) }\end{array}$ \\
\hline \multicolumn{13}{|c|}{ Ephippidae } \\
\hline & $\begin{array}{l}\text { Chaetodipterus faber } \\
\text { (Broussonet, 1782) }\end{array}$ & 4 & 2 & 2 & 0.02 & 0.02 & 0.02 & $32.0^{*}$ & $46.0^{*}$ & $\mathrm{ZB}$ & MM & $\begin{array}{l}\text { Krumme et al. } \\
(2004)\end{array}$ \\
\hline \multicolumn{13}{|c|}{ Haemulidae } \\
\hline & $\begin{array}{l}\text { Genyatremus luteus } \\
\text { (Bloch, 1790) }\end{array}$ & 5 & - & 5 & 0.02 & - & 0.05 & $23.0^{*}$ & $36.0^{*}$ & $\mathrm{ZB}$ & MM & $\begin{array}{l}\text { Barletta \& } \\
\text { Blaber (2007); } \\
\text { Krumme et al. } \\
(2004)\end{array}$ \\
\hline \multicolumn{13}{|c|}{ Loricariidae } \\
\hline & $\begin{array}{l}\text { Hypostomus punctatus } \\
\text { Valenciennes, } 1840\end{array}$ & 2 & 2 & - & 0.01 & 0.02 & - & $39.0^{*}$ & $50.0^{*}$ & DV & FS & $\begin{array}{l}\text { Froese \& Pauly } \\
\text { (2010) }\end{array}$ \\
\hline \multicolumn{13}{|c|}{ Mugilidae } \\
\hline & $\begin{array}{l}\text { Mugil incilis Hancock, } \\
1830\end{array}$ & 58 & 7 & 51 & 0.26 & 0.06 & 0.46 & $23.1 * *$ & $54.0^{* *}$ & - & MS & $\begin{array}{l}\text { Barros et al. } \\
(2011)\end{array}$ \\
\hline & Mugil sp. & 6 & - & 6 & 0.03 & - & 0.05 & $30.0^{* * *}$ & $47.0^{* *}$ & - & - & \\
\hline \multicolumn{13}{|c|}{ Pimelodidae } \\
\hline & $\begin{array}{l}\text { Brachyplatystoma } \\
\text { filamentosum } \\
\text { (Lichtenstein, 1819) }\end{array}$ & 221 & 192 & 29 & 0.99 & 1.72 & 0.26 & $2.0^{* * *}$ & $188.0^{* * *}$ & PV & FM & $\begin{array}{l}\text { Espírito Santo } \\
\text { et al. (2005); } \\
\text { Barthem \& } \\
\text { Goulding } \\
\text { (1997) }\end{array}$ \\
\hline & $\begin{array}{l}\text { Brachyplatystoma } \\
\text { platynemum } \\
\text { Boulenger, } 1898\end{array}$ & 67 & 10 & 57 & 0.30 & 0.09 & 0.51 & $12.0^{* * *}$ & $66.0^{* * *}$ & PV & FS & $\begin{array}{l}\text { Froese \& Pauly } \\
\text { (2010) }\end{array}$ \\
\hline & $\begin{array}{l}\text { Brachyplatystoma } \\
\text { rousseauxii } \\
\text { (Castelnau, 1855) }\end{array}$ & 8887 & 5147 & 3740 & 39.98 & 46.15 & $\begin{array}{c}33.7 \\
7\end{array}$ & $10.0^{* * *}$ & $170.0^{* * *}$ & PV & FM & $\begin{array}{l}\text { Espírito Santo } \\
\text { et al. (2005); } \\
\text { Barthem \& } \\
\text { Goulding } \\
\text { (1997) }\end{array}$ \\
\hline & $\begin{array}{l}\text { Hypophthalmus } \\
\text { marginatus } \\
\text { Valenciennes, } 1840\end{array}$ & 47 & 21 & 26 & 0.21 & 0.19 & 0.23 & $12.2^{* * *}$ & $44.0^{* * *}$ & $\mathrm{ZP}$ & FM & $\begin{array}{l}\text { Froese \& Pauly } \\
\text { (2010); Junk } \\
\text { (1985) }\end{array}$ \\
\hline & $\begin{array}{l}\text { Pimelodella cristata } \\
\text { (Müller \& Troschel, } \\
\text { 1848) }\end{array}$ & 7 & 4 & 3 & 0.03 & 0.04 & 0.03 & $10.0^{* * *}$ & $28.2^{* *}$ & $\mathrm{ZB}$ & FS & $\begin{array}{l}\text { Froese \& Pauly } \\
\text { (2010) }\end{array}$ \\
\hline & $\begin{array}{l}\text { Pinirampus pirinampu } \\
\text { (Spix \& Agassiz, } \\
\text { 1829) }\end{array}$ & 9 & 1 & 8 & 0.04 & 0.01 & 0.07 & $37.0^{* * *}$ & $67.0^{* * *}$ & PV & FS & $\begin{array}{l}\text { Froese \& Pauly } \\
\text { (2010) }\end{array}$ \\
\hline \multicolumn{13}{|c|}{ Polynemidae } \\
\hline & $\begin{array}{l}\text { Polydactylus } \\
\text { virginicus (Linnaeus, } \\
1758 \text { ) }\end{array}$ & 3 & 3 & - & 0.01 & 0.03 & - & $33.0^{* * *}$ & $50.0^{* *}$ & $\mathrm{ZB}$ & MM & $\begin{array}{l}\text { Barletta \& } \\
\text { Blaber (2007) }\end{array}$ \\
\hline \multicolumn{13}{|c|}{ Pristigasteridae } \\
\hline & $\begin{array}{l}\text { Pellona flavipinnis } \\
\text { (Valenciennes, 1837) }\end{array}$ & 509 & 273 & 236 & 2.29 & 2.45 & 2.13 & $4.0^{* * *}$ & $72.7^{* * *}$ & PV & FM & $\begin{array}{l}\text { Froese \& Pauly } \\
\text { (2010); Léopold } \\
\text { (2004) }\end{array}$ \\
\hline \multicolumn{13}{|c|}{ Sciaenidae } \\
\hline & $\begin{array}{l}\text { Ctenosciaena } \\
\text { gracilicirrhus } \\
\text { (Metzelaar, 1919) }\end{array}$ & 633 & 93 & 540 & 2.85 & 0.83 & 4.88 & $15.0^{*}$ & $83.1^{*}$ & $\mathrm{ZB}$ & MS & $\begin{array}{l}\text { Froese \& Pauly } \\
\text { (2010) }\end{array}$ \\
\hline & $\begin{array}{l}\text { Cynoscion acoupa } \\
\text { (Lacepède, 1801) }\end{array}$ & 96 & 65 & 31 & 0.43 & 0.58 & 0.28 & $32.0^{*}$ & $99.0^{*}$ & $\begin{array}{l}\text { PV/ } \\
\text { ZB }\end{array}$ & MM & $\begin{array}{l}\text { Krumme et al. } \\
\text { (2004) }\end{array}$ \\
\hline & $\begin{array}{l}\text { Macrodon ancylodon } \\
\text { (Bloch \& Schneider, } \\
\text { 1801) }\end{array}$ & 88 & 5 & 83 & 0.40 & 0.04 & 0.75 & $14.0^{*}$ & $53.0^{*}$ & $\begin{array}{l}\mathrm{PV} / \\
\mathrm{ZB}\end{array}$ & MM & $\begin{array}{l}\text { Camargo \& } \\
\text { Isaac (2004); } \\
\text { Krumme et al. } \\
(2004)\end{array}$ \\
\hline & $\begin{array}{l}\text { Micropogonias } \\
\text { furnieri (Desmarest, } \\
1823 \text { ) }\end{array}$ & 509 & 67 & 442 & 2.29 & 0.60 & 3.99 & $13.5^{*}$ & $89.0^{*}$ & ZB & MM & $\begin{array}{l}\text { Krumme et al. } \\
(2004)\end{array}$ \\
\hline
\end{tabular}


Table 1. Cont

\begin{tabular}{|c|c|c|c|c|c|c|c|c|c|c|c|c|}
\hline \multirow[t]{2}{*}{ Family } & \multirow[t]{2}{*}{ Scientific name } & \multicolumn{3}{|c|}{ Absolute Frequency } & \multicolumn{3}{|c|}{ Relative Frequency } & \multicolumn{2}{|c|}{$\begin{array}{l}\text { Length Ranges } \\
(\mathrm{cm})\end{array}$} & \multicolumn{3}{|c|}{ Guilds } \\
\hline & & Total & Rainy & Dry & Total & Rainy & Dry & Min & Max & FMFG & EUFG & Source \\
\hline \multicolumn{13}{|l|}{ Sciaenidae } \\
\hline & $\begin{array}{l}\text { Nebris microps } \\
\text { Cuvier, } 1830\end{array}$ & 4 & 2 & 2 & 0.02 & 0.02 & 0.02 & $27.0^{*}$ & $34.0^{*}$ & ZB & MM & $\begin{array}{l}\text { Froese \& Pauly } \\
\text { (2010); Espírito } \\
\text { Santo et al. }\end{array}$ \\
\hline & $\begin{array}{l}\text { Plagioscion auratus } \\
\text { (Castelnau, 1855) }\end{array}$ & 1 & - & 1 & 0.00 & - & 0.01 & $35.0^{*}$ & $35.0^{*}$ & $\mathrm{PV}$ & FS & $\begin{array}{l}\text { Barros et al. } \\
\text { (2011); Froese } \\
\text { \& Pauly (2010) }\end{array}$ \\
\hline & $\begin{array}{l}\text { Plagioscion } \\
\text { squamosissimus } \\
\text { (Heckel, 1840) }\end{array}$ & 3879 & 2572 & 1307 & 17.45 & 23.06 & $\begin{array}{c}11.8 \\
0\end{array}$ & $7.0^{*}$ & $90.0^{*}$ & PV & FM & $\begin{array}{l}\text { Espírito Santo } \\
\text { et al. (2005); } \\
\text { Léopold (2004) }\end{array}$ \\
\hline & $\begin{array}{l}\text { Plagioscion } \\
\text { surinamensis (Bleeker, } \\
\text { 1873) }\end{array}$ & 371 & 240 & 131 & 1.67 & 2.15 & 1.18 & $10.0^{*}$ & $140.0^{*}$ & PV & FM & $\begin{array}{l}\text { Froese \& Pauly } \\
\text { (2010) }\end{array}$ \\
\hline \multicolumn{13}{|l|}{ Scombridae } \\
\hline & $\begin{array}{l}\text { Acanthocybium } \\
\text { solandri (Cuvier, } \\
1832 \text { ) }\end{array}$ & 2 & 2 & - & 0.01 & 0.02 & - & $37.0^{* * *}$ & $37.0^{* *}$ & PV & MM & $\begin{array}{l}\text { Froese \& Pauly } \\
\text { (2010); Espírito } \\
\text { Santo et al. } \\
\text { (2005) }\end{array}$ \\
\hline & $\begin{array}{l}\text { Scomberomorus } \\
\text { brasiliensis Collette, } \\
\text { Russo \& Zavala- } \\
\text { Camin, } 1978\end{array}$ & 1 & 1 & - & 0.00 & 0.01 & - & $44.0^{* * *}$ & $44.0^{* * *}$ & PV & MM & $\begin{array}{l}\text { Froese \& Pauly } \\
\text { (2010); Espírito } \\
\text { Santo et al. } \\
\text { (2005) }\end{array}$ \\
\hline \multicolumn{13}{|l|}{ Sternopygidae } \\
\hline & $\begin{array}{l}\text { Eigenmannia } \\
\text { virescens } \\
\text { (Valenciennes, 1836) }\end{array}$ & 3 & 3 & - & 0.01 & 0.03 & - & $25.5^{* * *}$ & $35.7 * *$ & ZB & FS & $\begin{array}{l}\text { Barletta \& } \\
\text { Blaber (2007) }\end{array}$ \\
\hline Tetraodontidae & $\begin{array}{l}\text { Colomesus psittacus } \\
\text { (Bloch \& Schneider, } \\
\text { 1801) }\end{array}$ & 1 & 1 & - & 0.00 & 0.01 & - & $45.0^{*}$ & $45.0^{*}$ & $\mathrm{ZB}$ & ES & $\begin{array}{l}\text { Giarrizzo et al. } \\
\text { (2010); } \\
\text { Krumme et al. } \\
\text { (2007); Barletta } \\
\text { \& Blaber } \\
\text { (2007); } \\
\text { Krumme et al. } \\
\text { (2004) }\end{array}$ \\
\hline \multicolumn{13}{|l|}{ Trichiuridae } \\
\hline & $\begin{array}{l}\text { Trichiurus lepturus } \\
\text { Linnaeus, } 1758\end{array}$ & 3 & 1 & 2 & 0.01 & 0.01 & 0.02 & $44.0^{*}$ & $92.0^{*}$ & PV & MS & $\begin{array}{l}\text { Barletta \& } \\
\text { Blaber (2007) }\end{array}$ \\
\hline \multicolumn{13}{|l|}{ Not identified } \\
\hline & Rays & 146 & 22 & 124 & 0.66 & 0.20 & 1.12 & $11.0^{*}$ & $180.0^{*}$ & - & - & \\
\hline & Caldeirada & 19 & 14 & 5 & 0.09 & 0.13 & 0.05 & $17.0^{* *}$ & $43.0^{* * *}$ & - & - & \\
\hline & Jurubeba & 102 & 7 & 95 & 0.46 & 0.06 & 0.86 & $15.0^{* * *}$ & $46.0^{* * *}$ & - & - & \\
\hline & Jururema & 17 & 17 & - & 0.08 & 0.15 & - & $20.0^{* * *}$ & $35.0^{* * *}$ & - & - & \\
\hline & Lambe-lambe & 12 & 9 & 3 & 0.05 & 0.08 & 0.03 & $10.0^{*}$ & $48.0^{*}$ & - & - & \\
\hline & Tuiuiu & 15 & 12 & 3 & 0.07 & 0.11 & 0.03 & $29.0^{*}$ & $44.0^{*}$ & - & - & \\
\hline Total & & 22228 & 11153 & 11075 & 100 & 100 & 100 & - & - & - & - & \\
\hline
\end{tabular}

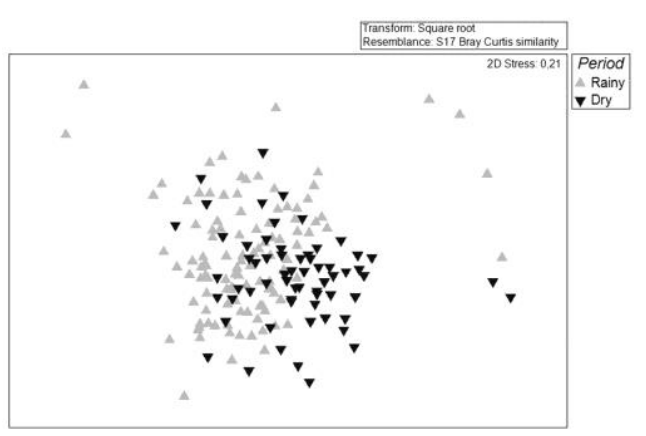

Fig. 2. Results of a MDS ordination analysis with the rainy and dry seasons as factors.

\section{Ecological Guilds}

Overall, the largest environmental guild in terms of the number of species (Fig. 3a) was that of the marine migrants $(26.8 \%)$, followed by the estuarine species (24.4\%). The same pattern was observed during the rainy season. In the dry season
(Fig. 3a), however, estuarine species dominated $(27.3 \%)$, followed by marine migrants $(21.2 \%)$, freshwater stragglers $(21.2 \%)$, and freshwater migrants $(21.2 \%)$.

In contrast, freshwater migrants predominated $(64.1 \%)$ in terms of the number of individuals captured during the study period (Fig. 3b), followed by estuarine species $(23.3 \%)$. The same pattern was observed in both seasons, although the percentage of marine (visitors and migrants) and estuarine species increased during the dry season in comparison with the rainy season (Fig. 3b).

The zoobenthivores (ZB) were the richest feeding guild in terms of the number of species (43.6\%), followed by the piscivores, PV (30.8\%). These two guilds also predominated in both seasons, representing $41.7 \%$ and $30.6 \%$ of the species, respectively, in the rainy season and $38.7 \%$ and $32.3 \%$ in the dry. The species that feed on fish and zoobenthos (PV/ZB) were also prominent in both seasons (Fig. 4a). In terms of the number of 
individuals captured, however, the piscivores predominated $(64.8 \%)$ throughout the study period, representing $76.5 \%$ of the catch during the rainy season and $52.5 \%$ during the dry season (Fig. 4b).

\section{Body Length and Catch Probability}

The standard length (SL) of the B. vaillantii specimens ranged from 2.6 to $91.7 \mathrm{~cm}$, with a high frequency $(83 \%)$ of individuals between 20 and $40 \mathrm{~cm}$. The estimated catch probability $\left(\mathrm{P}_{50}\right)$ for this species was $25.27 \mathrm{~cm}$ (Fig. 5). A similar pattern was recorded

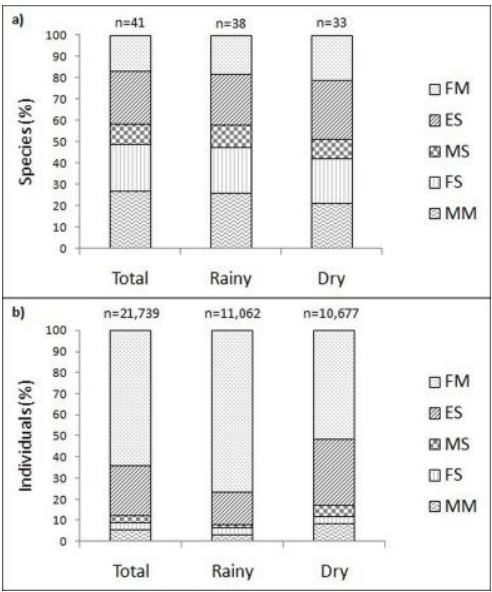

Fig. 3. Percentage of (a) species and (b) individuals present in the bycatch of the $B$. vaillantii trawler fleet by environmental guild. Marine Stragglers (MS), Marine Migrants (MM), Freshwater Stragglers (FS), Freshwater Migrants (FM), Estuarine Species (ES), and numbers (n) of (a) species and (b) individuals. for $B$. rousseauxii (SL: 9.5-153.4 cm), with approximately $36 \%$ of individuals in the $40-50 \mathrm{~cm}$ class (Fig. 5). The $\mathrm{P}_{50}$ value estimated for $B$. rousseauxii was $35.96 \mathrm{~cm}$. In the case of $P$. squamosissimus, total length (TL) ranged from 7 to $90 \mathrm{~cm}$, with most individuals in the $50-60 \mathrm{~cm}$ class and $\mathrm{P}_{50}$ estimated as $43.94 \mathrm{~cm}$ (Fig. 5). Similarly, $S$. herzbergii presented fork lengths (FL) between 10 and $130 \mathrm{~cm}$ but primarily between 30 and $50 \mathrm{~cm} \mathrm{(42 \% ).}$ The $\mathrm{P}_{50}$ value for this species was $40.34 \mathrm{~cm}$ (Fig. 5).

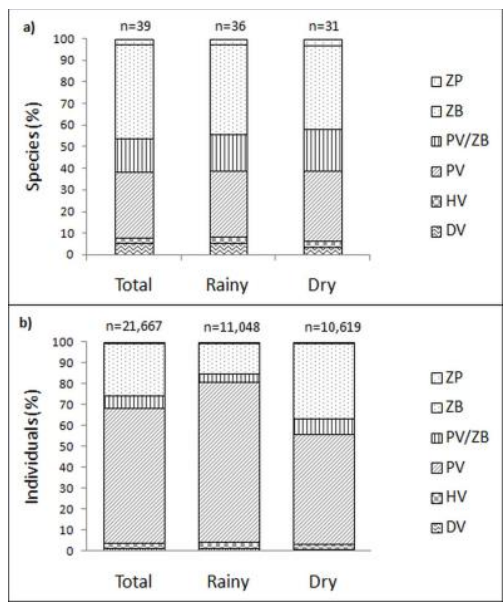

Fig. 4. Percentage of (a) species and (b) individuals present in the bycatch of the $B$. vaillantii trawler fleet by feeding guild. Zooplanktivore (ZP), Zoobenthivore (ZB), Piscivore/Zoobenthivore (PV/ZB), Piscivore (PV), Herbivore (HV), Detritivore (DV), and numbers (n) of (a) species and (b) individuals.

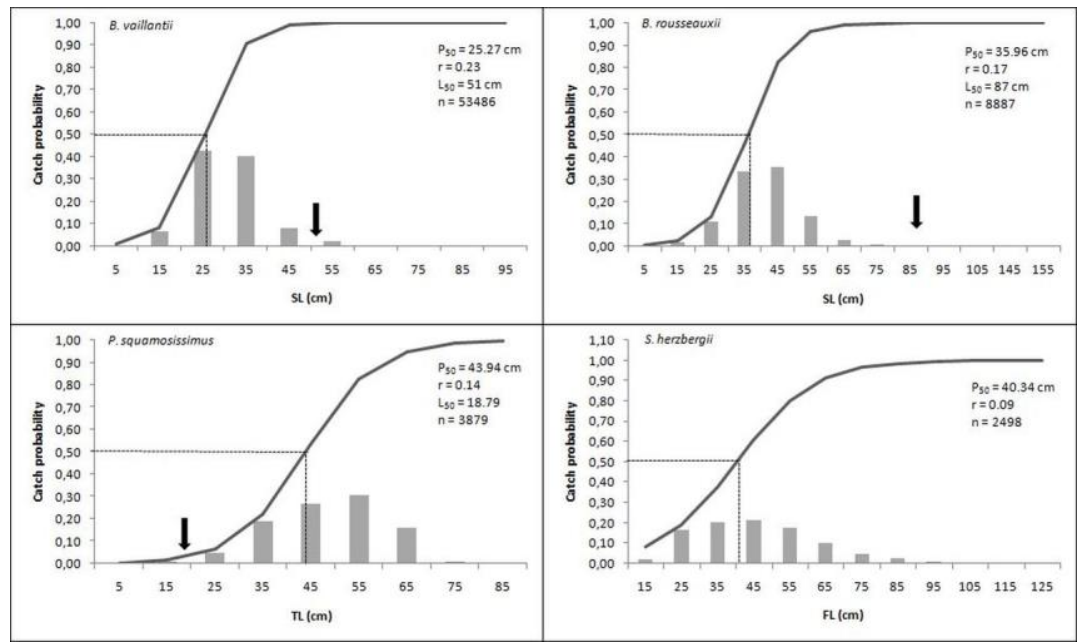

Fig. 5. Catch probability estimated for $B$. vaillantii and the principal bycatch species. The dashed lines indicate the length at which catch probability is $50 \%\left(\mathrm{P}_{50}\right)$, the arrows indicate the estimated length of first sexual maturity $\left(\mathrm{L}_{50}\right), \mathrm{r}$ is the slope of the logistic curve, and $\mathrm{n}$ is the number of individuals measured. The $\mathrm{L}_{50}$ values for $B$. vaillantii, $B$. rousseauxii and $P$. squamosissimus were obtained from Vásquez et al. (2009), Fabré et al. (2000) and Barbosa et al. (2012), respectively. 


\section{Discussion}

In recent decades, many technological advances have been made in an effort to reduce bycatch, but the capture and discarding of unwanted species remains a major problem for commercial fisheries worldwide (FAO, 2010). Industrial trawling for shrimp and demersal finfish represents more than half of the overall estimate of discarded bycatch (KELLEHER, 2005).

The industrial trawling fleets of the coast of northern Brazil operate primarily in the mouth of the Amazon River. These operations target primarily $B$. vaillantii (pair trawling), and pink (Farfantepenaeus subtilis, $F$. brasiliensis, and $F$. notialis) and white shrimp (Litopenaeus schimitti), using double rigs (PETRERE et al., 2007). In both cases, undersized specimens of the target species and any fish with no commercial value are discarded as bycatch (BARTHEM; GOULDING, 1997; BARTHEM, 2000).

During the peak of the industrial harvesting of B. vaillantii, in the 1970s, approximately $30 \%$ of the catch was rejected (ARAÚJO-LIMA; RUFFINO, 2003). In 1979, $20 \%$ of the total catch of this species was discarded, representing approximately 3,253 tons or 11 million specimens (DIAS-NETO et al., 1985). The results of the present study indicate that the fish bycatch of the industrial laulao trawler fleet in the Amazon Estuary is approximately $29 \%$ of the total catch (individuals). The number of taxa recorded in this study (52) was similar to those recorded in studies of the bycatch of shrimp trawling operations (SANTOS et al., 1998; CASTRIOTA et al., 2001; VIANNA et al., 2004; GOMES; CHAVES, 2006; BRANCO; VERANI, 2006).

The three principal families in terms of both species richness and the percentage of individuals captured were the Ariidae, Pimelodidae, and Sciaenidae, which are the most diverse within the study region (BARTHEM, 1985). Species representing the principal bycatch families in the present study are commercially important in northern Brazil (ESPÍRITO SANTO et al., 2005) and represent approximately $14 \%$ of the total catch landed in Pará State, the equivalent of approximately 13,400 tons (SEPAQ, 2008). Of these species, $B$. rousseauxii, $P$. squamosissimus, and S. herzbergii together represent approximately $69 \%$ of the total catch and were considered to be consistent and essential components of the bycatch of the industrial $B$. vaillantii fleet. Pinheiro and Lucena Frédou (2004) have recorded similar results for the laulao trawling fleet in the Amazon Estuary, although the catfish Sciades couma was the dominant bycatch species in this case, followed by $B$. rousseauxii and $P$. squamosissimus.

The composition of the bycatch was similar between seasons, as confirmed by MDS and
ANOSIM, with approximately $71 \%$ of the taxa occurring in both periods. There was some variation in catch volume, however, with limnic species, such as $B$. rousseauxii and $P$. squamosissimus, more common during the rainy season, and marine species (e.g., $S$. herzbergii) predominating during the dry season. This pattern is typical of the Amazon Estuary, in which freshwater fish species predominate during the period of maximum fluvial discharge (rainy season), whereas marine species are more common during the dry season (BARTHEM, 1985). This difference occurs because the increased discharge during the rainy season tends to displace the more saline water of marine origin (EGLER; SCHWASSMANN, 1962), allowing freshwater species, such as $B$. rousseauxii and $P$. squamosissimus, to range into coastal areas. In the dry season, in contrast, the intrusion of seawater produces an increase in salinity in the inner portion of the estuary, forcing the freshwater species further upriver into the Amazon and Tocantins basins and enabling marine species, such as $S$. herzbergii, to range into the estuary (BARTHEM, 1985).

In terms of the number of species, the marine migrants and estuarine species were the most diverse environmental guilds. However, freshwater migrants were dominant in numbers, due primarily to the abundance of $B$. rousseauxii and $P$. squamosissimus. In the case of the feeding guilds, the zoobenthivore group was predominant in terms of the number of species, while the piscivores predominated in relative abundance, representing approximately $65 \%$ of the total individuals captured.

Estuarine substrates are ideal environments for many organisms due to the relatively rich deposits of organic matter and inorganic nutrients (MCLUSKY, 1989). Muddy sediments dominate the inner continental shelf of the Amazon estuary (BARRETO et al., 1975), providing a considerable abundance and diversity of resources (LOWEMCCONNELL, 1962) to a rich fauna of benthic invertebrates, which feed primarily on organic debris (DAY et al., 1989). As a result, a substantial variety of fishes that feed on these organisms are attracted to these environments (HARRISON; WHITFIELD, 2004), especially species with demersal habits, which show a high productivity in the region (ISAAC et al., 1998).

In the present study, trawling had a clear effect on populations by capturing large quantities of juveniles, as indicated by the numbers of small- (20-30 $\mathrm{cm})$ and medium-sized $(30-50 \mathrm{~cm})$ individuals in the catches. These captures primarily affected the stocks of $B$. rousseauxii and $B$. vaillantii. The catch of $B$. rousseauxii was composed primarily (99\%) of immature individuals. Approximately one-third (32\%) of the catch consists of young, non-migrating individuals $(<40 \mathrm{~cm})$, whereas $67 \%$ are young 
individuals with lengths between $40 \mathrm{~cm}$ and $80 \mathrm{~cm}$. Barthem (2000) has observed that $B$. rousseauxii juveniles and pre-adults (1-3 years) are common in the estuary, whereas adults are rare or absent. Alonso and Pirker (2005) have reported that large numbers of $B$. rousseauxii are harvested commercially before beginning their reproductive cycle.

Although the fish in question belonged to the target species, most of the catch $(98 \%)$ of B. vaillantii consisted of immature individuals. Approximately $4 \%$ of the total catch consisted of young, non-migrating individuals $(<20 \mathrm{~cm})$, whereas $14 \%$ were pre-adults with a length of $40-50 \mathrm{~cm}$. These smaller and younger individuals may be relatively common in estuaries, where Alonso and Pirker (2005) observed that $56 \%$ of the catches of commercial artisanal fisheries consisted of individuals with a FL of $20-40 \mathrm{~cm}$. The high mortality of juvenile $B$. vaillantii below marketable size appears to be related to the small mesh size used by the local fisheries (BARTHEM; GOULDING, 1997).

Alonso and Pirker (2005) have characterised the Amazon estuary as a nursery for both $B$. vaillantii and $B$. rousseauxii. The abundant food and space in the estuary, in addition to the low number of predators, allows rapid growth during the first years of life and increases the probability of survival. The upstream migrations, which involve distances greater than 3,000 $\mathrm{km}$, begin when the individuals are two years old (BARTHEM; GOULDING, 1997).

In $P$. squamosissimus, by contrast, the catch consisted primarily $(99 \%)$ of large adults. Given that this species is of commercial value, it is probable that this catch is included in the official statistics. The elimination of these individuals from the population may, nevertheless, have an equally marked impact on recruitment.

Mortality from fishery operations tends to be more harmful to K-strategist species (ALVERSON et al., 1994), as is the case for B. vaillantii. This species is relatively long-lived, with a life expectancy of 22 years, grows slowly $(0.13 \mathrm{~cm}$ per year) (ALONSO; PIRKER 2005), and matures late. Alonso and Pirker (2005) concluded that $B$. rousseauxii is being harvested at its maximum limit of exploitation. Other vulnerable species include rays, which are commonly caught as bycatch (JICA, 1998), and ariid catfishes, which have well-developed parental care, such as oral incubation of eggs and embryos (REIS, 1986; YAÑEZ-ARANCIBIA; SÀNCHEZ-GIL， 1988; BARBIERI et al., 1992; CHAVES, 1994).

A number of different mitigating strategies can be considered for the bycatch problem. Hall (1999) emphasises the need for increased selectivity and the prohibition of fishing in certain areas and seasons. Selectivity can be enhanced by increasing the mesh size and by the adoption of Bycatch Reduction Devices (BRDs).

One other option is the deployment of Marine Protected Areas (MPAs). According to the Government of Canada (2005), these areas can restore the balance of marine ecosystems and provide economic, social, and cultural benefits. These MPAs may be particularly effective as long-term strategies for migratory species and those with a long life cycle and slow maturation, such as $B$. vaillantii and $B$. rousseauxii, by protecting the habitats used during the most vulnerable phases of the life cycle, thus guaranteeing higher recruitment rates. The protection of areas characterised by high bycatch rates, especially in relationship to species vulnerable to overfishing, is also recommended.

Trawling is currently prohibited in the Amazon and Pará estuaries in the area between $00^{\circ} 05^{\prime}$ $\mathrm{N}$ and $48^{\circ} 00^{\prime} \mathrm{W}$ (Federal decree no. 6 of 07/06/04). However, this measure appears to have been ineffective for the protection of small individuals, given that a large proportion of the bycatch still consists of juveniles. In the light of this finding, the expansion of the prohibited zone during the dry season, when non-migrating juveniles are most vulnerable, would be one additional effective measure.

Bottom trawling directed to laulao remains the focus of many social conflicts due to the impact of the fishing gear and the great fishing power of the fishery (ISAAC et al., 2011). Initiatives to decrease the negative effect of this fishery have not improved the situation over time. This lack of improvement is due primarily to the limitations of on-board space, the low refrigeration capacity of the fleet, the disproportionate relationships involving the price per kilogram of the target species and of the non-target species, and the time taken for selection and separation of the bycatch individuals (IDESP, 1989; PAIVA, 1997; ISAAC; BRAGA, 1999).

\section{ACKNOWLEDGEMENTS}

We thank the Acquamazon Institute for providing the database that facilitated this study, CAPES for the award of a scholarship, Dr. Ronaldo Barthem for his contribution to the previous version of the manuscript, and Dr. Thierry Frédou for assistance with the statistical analysis.

\section{REFERENCES}

ALONSO, J. C.; PIRKER, L. E. M. Dinâmica populacional e estado atual da exploração da piramutaba e da dourada. In: FABRÉ, N. N.; BARTHEM, R. B. (Orgs.). O manejo da pesca dos grandes bagres migradores: piramutaba e dourada no eixo Solimões-Amazonas. Manaus: ProVárzea/IBAMA, 2005. cap. 3. (Coleção Documentos Técnicos: Estudos Estratégicos). 
ALVERSON, D. L.; FREEBERG, M. H.; MURAWSKI, S. A.; POPE, J. G. A global assessment of fisheries bycatch and discards. FAO Fish. Tech. Pap., n. 339, 1994.

ALVERSON, D. L.; HUGHES, S. E. Bycatch: from emotion to effective natural resource management. Rev. Fish Biol. Fish., v. 6, n. 4, p. 443-462, 1996.

ARAÚJO-LIMA, C. A. R. M.; RUFFINO, M. L. Migratory fishes of the Brazilian Amazon. In: CAROLSFIELD, J.; HARVEY, B.; ROSS, C.; BAER, A. (Orgs.). Migratory fishes of South America: Biology, Fisheries and Conservation Status. Canada: IDRC/World Bank, 2003. cap 6.

BARBIERI, L. R.; SANTOS, R. P. J.; ANDREATA, J. V. Reproductive biology of the marine catfish, Genidens genidens (Siluriformes, Ariidae), in the Jacarepaguá Lagoon system, Rio de Janeiro, Brazil. Environ. Biol. Fishes, v. 35, n. 1, p. 23-35, 1992.

BARBOSA, N. D. ROCHA, R. M.; LUCENA FRÉDOU, F. The reproductive biology of Plagioscion squamosissimus (Heckel, 1840) in the Pará River estuary (Amazon Estuary). J. Appl. Ichthyol., v. 28, n. 5, p. 800-805, 2012.

BARLETTA, M.; BLABER, S. J. M. Comparison of fish assemblages and guilds in tropical habitats of the Embley (Indo-West Pacific) and Caeté (Western Atlantic) estuaries. Bull. Mar. Sci., v. 80, n. 3, p. 647-680, 2007.

BARRETO, L. A.; MILLIMAN, J. D.; AMARAL, C. A. B.; FRANCISCONI, O. Northern Brazil. In: MILLIMAN, J. D.; SUMMERHAYES, C. P. (Ed.). Upper continental margin sedimentation off Brazil. Contrib. Sedimentol., v. 4, p. 11-43, 1975.

BARROS, D. F.; TORRES, M. F.; LUCENA FRÉDOU, F. A ictiofauna do estuário de São Caetano de Odivelas e Vigia (Pará, Estuário Amazônico). Biota Neotrop., v. 11, n. 2, p. 367-373, 2011.

BARTHEM, R. B. Ocorrência, distribuição e biologia dos peixes da Baía de Marajó, estuário amazônico. Bol. Mus. Para. Emílio Goeldi, Sér. Zool., v. 2, n. 1, p. 49-69, 1985.

BARTHEM, R. B. Situação do manejo das pescarias dos grandes bagres amazônicos do Brasil. In: FAO (Food and Agriculture Organization of The United Nations). Informe del Taller Regional sobre Manejo de las Pesquerias de Bagres Migratorios del Amazonas. Roma: FAO/COPESCAL, 2000. p. 37-60. (Programa de Cooperación Gubernamental FAO/Noruega).

BARTHEM, R. B.; GOULDING, M. The Catfish Connection: Ecology, Migration, and Conservation of Amazon Predators. New York: Columbia University Press, 1997. 144 p.

BARTHEM, R. B.; PETRERE, M. JR. Fisheries and Population Dynamics of the Freshwater catfish Brachyplatystoma vaillantii in the Amazon Estuary. In: ARMANTROUT, N. B.; WOLOTIRA, R. J. (Eds.). Conditions of the Worlds Aquatic Habitats. New Delhi: Oxford \& IBH Publishing CO. PVT. LTD, 1995. p. 329 - 340. (Proceedings of the World Fisheries Congress - Theme 1).

BRANCO, J. O.; VERANI, J. R. Análise quali-quantitativa da ictiofauna acompanhante na pesca do camarão setebarbas, na Armação Itapocoroy, Penha, Santa Catarina. Rev. Bras. Zool., v. 23, n. 2, p. 381-291, 2006.

CAMARGO, M.; ISAAC, V. J. Food categories reconstruction and feeding consumption estimates for the sciaenid Macrodon ancylodon (Bloch \& Schneider), and the congeneric fishes Stellifer rastrifer (Jordan) and Stellifer naso (Jordan) (Pisces, Perciformes) in the Caeté estuary, northern coast of Brazil. Rev. Bras. Zool., v. 21, n. 1, p. 85-89, 2004.

CAMARGO, M.; ISAAC, V. J. Population structure of fish fauna in the estuarine area of Caeté River, Bragança, Pará, Brazil. Acta Sci., v. 20, n. 2, p. 171-177, 1998.

CASEY, J. M.; MYERS, M. A. Near extinction of a large, widely distributed fish. Science, v. 281, n. 5377, p. 690692, 1998.

CASTRIOTA, L.; CAMPAGNUOLO, S.; ANDALORO, F. Shrimp trawl Fishery By-catch in the Straits of Sicily (Central Mediterranean Sea). NAFO SCR, doc. 01/113, serial ${ }^{\circ} \mathrm{N} 4501,2001$.

CHAVES, P. T. C. A incubação de ovos e larvas em Genidens genidens (Valenciennes) (Siluriformes, Ariidae) da Baía de Guaratuba, Paraná, Brasil. Rev. Bras. Zool., v. 11, n. 4, p. 641-648, 1994.

CLARKE, K. R. Non-parametric multivariate analyses of changes in community structure. Aust. J. Ecol., v. 18, n. 1, p. 117-143, 1993.

CLUCAS, I. A study of the options for utilization of bycatch and discards from marine capture fisheries. Rome: FAO, 1997. 59 p.

DAJOZ, R. Ecologia Geral. São Paulo: Vozes, 1973. 472 p.

DAY, J. W. JR.; HALL, C. A. S.; KEMP, W. M.; YÁÑEZARANCIBIA, A. Estuarine ecology. NewYork: John Wiley \& Sons, 1989. $558 \mathrm{p}$

DIAS NETO, J.; DAMASCENO, J. F. G. \& PONTES, A. C. Biologia e pesca da piramutaba, Brachyplatystoma vailantii Valenciennes, na região Norte do Brasil. Brasília: SUDEPE, 1985. (Série Documentos Técnicos $\left.n^{\circ} 35\right)$.

EGLER, W. A.; SCHWASSMANN, H. O. Limnological studies in the Amazon Estuary. Bol. Mus. Para. Emilio Goeldi, v. 1, p. 2-25, 1962. Publicação avulsa.

ELLIOT, M.; WHITFIELD, A. K.; POTTER, I. C.; BLABER, S. J. M.; CYRUS, D. P.; NORDLIE, F. G. \& HARRISON, T. D. The guild approach to categorizing estuarine fish assemblages: a global review. Fish Fish., v. 8, n. 3, p. $241-268,2007$.

ESPÍRITO SANTO, R. V.; ISAAC, V. J.; SILVA, L. M. A.; MARTINELLI, J. M.; HIGUCHI, H.; SAINT-PAUL, U. Peixes se camarões do litoral bragantino, Pará Brasil. Belém: MADAM, 2005. 268 p.

FABRÉ, N. N.; ALONSO, J. C. Recursos ícticos no Alto Amazonas: Sua importância para as populações ribeirinhas. Bol. Mus. Para. Emílio Goeldi, Sér. Zool., v. 14, n. 1, p. 19-55, 1998.

FABRÉ, N. N.; DONATO, J. C.; ALONSO, J. C. (Eds.) Bagres de la Amazonia Colombiana: un recurso sin fronteras. Bogotá: SINCHI, 2000. 252 p.

FAO (Food and Agriculture Organization of The United Nations). The state of world fisheries and aquaculture 2010. Roma: FAO, 2010. 197 p.

FROESE, R.; PAULY, D. (Eds.). Fishbase. World wide web electronic publication. Version 11/2010. Available at: .

GIARRIZZO, T.; KRUMME, U.; WOSNIOK, W. Sizestructure migration and feeding patterns in the banded puffer fish Colomesus psittacus (Tetraodontidae) from north brazilian mangrove creeks. Mar. Ecol. Prog. Ser., v. 419, p. $157-170,2010$. 
GIARRIZZO, T.; SAINT-PAUL, U. Ontogenetic and seasonal shifts in the diet of the pemecou sea catfish Sciades herzbergii (Siluriformes: Ariidae), from a macrotidal mangrove creek in the Curuçá estuary, northern Brazil. Rev. Biol. Trop., v. 56, n. 2, p. 861-873, 2008.

GOMES, I. D.; CHAVES, P. T. Ictiofauna integrante da pesca de arrasto camaroeiro no litoral sul do estado do Paraná, Brasil. Bioikos, v. 20, n. 1, p. 9-13, 2006.

GOVERNMENT OF CANADA. Canada's Federal Marine Protected Areas Strategy. Ottawa: Government of Canada, 2005. $18 \mathrm{p}$.

HALL, S. J. The effects of fishing on marine ecosystems and communities. Oxford: Blackwell Science, 1999. (Fish Biology and Aquatic Resources Series 1).

HALL, S. J.; MAINPRIZE, B. M. Managing by-catch and discards: how much progress are we making and how can we do better? Fish Fish., v. 6, p. 134-155, 2005.

HARRISON, T. D.; WHITFIELD, A. K. A multi-metric fish index to assess the environmental condition of estuaries. J. Fish Biol., v. 65, p. 683-710, 2004.

IBAMA (Instituto Brasileiro do Meio Ambiente e dos Recursos Naturais Renováveis). Relatório estatístico da pesca - 2007. Brasil - Grandes Regiões e Unidades da Federação. Brasília - DF: IBAMA, 2007. 151 p.

IDESP (Instituto de Desenvolvimento Econômico, Social e Ambiental do Pará). Relatório de Pesquisa. A pesca no Pará: A sócio-economia da fauna acompanhante do camarão na costa norte do Brasil e a comercialização da pesca artesanal em Belém, Vigia e Bragança. Belém: IDESP/SECIRM, 1989. 116 p.

ISAAC, V. J. Fisheries by-catch in the northern coast of Brazil: an anthology of waste. In: CLUCAS, I.; TEUTSCHER, F. (Orgs.). Report and proceedings of FAO/DFID expert consultation on bycatch utilization in tropical fisheries. United Kingdom: FAO/DFID Expert Consultation, 1998. p. 273-294.

ISAAC, V. J., BRAGA, T. M. P. Rejeição de pescado nas pescarias da costa norte do Brasil. Arq. Cien. Mar., v. 32, p. 39-54, 1999.

ISAAC, V. J.; RUFFINO, M. L.; MCGRATH, D. In search of a new approach to fisheries management in the middle Amazon region. In: FUNK, F.; HEIFTETS, J.; IANELLES, J.; POWER, J.; QUINN, T. SCHWEIGERT, J.; SULLIVAN, P.; AHANG, C. I. (Eds.). Fishery Stock Assessment Models for the $21^{\text {st }}$ Century. Anchorage: Alaska Sea Grant College Program, 1998. 889-902 p.

ISAAC, V. J.; SANTO, R. V. E.; BENTES, B.; MOURAO, K. R. M.; FREDOU, T.; LUCENA FREDOU, F. Uma avaliação interdisciplinar dos sistemas de produção pesqueira do estado do Pará, Brasil. In: HAIMOVICI, M. (Org.). Sistemas pesqueiros marinhos e estuarinos do Brasil. Rio Grande: FURG, 2011. p. 8-24.

JICA (JAPAN INTERNATIONAL COOPERATION AGENCY). Draft final report for the fishery resources study of the Amazon and Tocantins rivers mouth areas in the federative republic of Brazil. Tokyo: JICA/Sanyo Techno Marine, 1998. 334 p.

JUNK, W. J. Temporary fat storage, an adaptation of some fish species to the water level fluctuations and related environmental changes of the Amazon river. Amazoniana, v. 9, n. 3, p. 315-351, 1985.
KELLEHER, K. Discards in the world's marine fisheries: An Update. FAO Fish. Tech. Pap., n. 470, 2005.

KENELLY, S. J. The issue of bycatch in Australia's demersal trawls fisheries. Rev. Fish Biol. Fish., v. 5, n. 2, p. 213-234, 1995.

KRUMME, U.; BRENNER, M.; SAINT-PAUL, U. Springneap cycle as a major driver of temporal variations in feeding of intertidal fishes: Evidence from the sea catfish Sciades herzbergii (Ariidae) of equatorial West Atlantic mangrove creeks. J. Exp. M. Biol. Ecol., v. 367, p. 9199, 2008.

KRUMME, U.; KEUTHEN, H.; SAINT-PAUL, U.; VILLWOCK, W. Contribution to the feeding ecology of the banded puffer fish Colomesus psittacus (Tetraodontidae) in north Brazilian mangrove creeks. Braz. J. Biol., v. 67, n. 3, p. 383-392, 2007.

KRUMME, U.; SAINT-PAUL, U.; ROSENTHAL, H. Tidal and diel changes in the structure of a nekton assemblage in small intertidal mangrove creeks in northern Brazil. Aquat. Living Resour., v. 17, p. 215-229, 2004.

LÉOPOLD, M. Guide des poissons de mer de Guyane. Cayenne: IFREMER, 2004. $126 \mathrm{p}$

LEWISON, R. L.; CROWDER, L. B.; READ, A. J.; FREEMAN, S. A. Understanding impacts of fisheries bycatch on marine megafauna. Trends Ecol. Evol., v. 19, n. 11, p. 598-604, 2004

LOWE-MCCONNELL, R. H. The fishes of the British Guiana Continental Shelf, Atlantic coast of South America, with notes on their natural history. J. Linn. Soc., Zool., v. 44, n. 301, p. 669-700, 1962.

MARTORANO, L. G.; PEREIRA, L. C.; CEZAR, E. G. M. \& PEREIRA, I. C. B. Estudos climáticos do Estado do Pará, classificação climática (Köppen) e deficiência hídrica (Thornthwhite Mather). Belém: SUDAM/EMBRAPA, 1993. 53 p.

MCLUSKY, D. The estuarine ecosystem. London: Chapman \& Hall, 1989. 215 p.

MDIC (Ministério do Desenvolvimento, Indústria e Comércio Exterior); ALICEWEB (Sistema de Análise das Informações de Comércio Exterior da Secretaria de Comércio Exterior - SECEX). Análise do período de 2007 a 2010. Available at: . Accessed Sept. $10,2011$.

MEES, G. F. The Auchenipteridae and Pimelodidae of Suriname (Pisces, Nematognathi). Zool. Verh., n. 132, p. 1-256, 1974.

MENDES, F. L. S.; BARTHEM, R. B. Hábitos alimentares de bagres marinhos (Siluriformes: Ariidae) do estuário amazônico. Amazônia: Ci. \& Desenv., v.5, n. 10, p. 153-166, 2010

MORAES, B. C.; COSTA, J. M. N.; COSTA, A. C. L.; COSTA, M. H. Variação espacial e temporal da precipitação no Estado do Pará. Acta Amaz., v. 35, n. 2, p. 207-214, 2005.

MPA (Ministério da Pesca e Aqüicultura). Boletim Estatístico da Pesca e Aqüicultura - Brasil 2010. Brasília - DF: MPA, 2012. 129 p.

MURRAY, J. D.; BAHEN, J. J.; RULIFSON, R. A. Management considerations for bycatch in the North Carolina and Southeast shrimp fishery. Fisheries, v. 17, n. 1, p. 21-26, 1992.

PAIVA, M. P. Recursos pesqueiros estuarinos e marinhos do Brasil. Fortaleza: UFC Edições, 1997. 286 p. 
PARENTE, V. M.; VIEIRA, E. F.; CARVALHO, A. R.; FABRÉ, N. N. A pesca e a economia da pesca de bagres no eixo Solimões-Amazonas. In: FABRÉ, N. N.; BARTHEM, R. B. (Orgs.). O manejo da pesca dos grandes bagres migradores: piramutaba e dourada no eixo Solimões-Amazonas. Manaus: ProVárzea/IBAMA, 2005. cap 4. (Coleção Documentos Técnicos: Estudos Estratégicos).

PETRERE, M. JR.; BATISTA, V. S.; FREITAS, C. E. C.; ALMEIDA, O. T.; SURGIK, A. C. S. Amazônia: Ambientes, recursos e pesca. In: PETRERE JÚNIOR, M. (Org.). O setor pesqueiro na Amazônia: Situação atual e tendências. Manaus: ProVárzea/IBAMA, 2007. p. 11-18.

PINHEIRO, L. S.; LUCENA FRÉDOU, F. Caracterização geral da pesca industrial desembarcada no Estado do Pará. Revista Científica da UFPA, v. 4, 2004.

REIS, E. G. Reproduction and feeding habits of the marine catfish Netuma barba (Siluriformes, Ariidae), in the estuary of Lagoa dos Patos, Brasil. Atlântica, 8, p. 35$55,1986$.

SAILA, S. B. Importance and assessment of discards in commercial fisheries. Rome: FAO, 1983. 62 p. (FAO Fish. Circ. n. 765)

SANTOS, M. C. F.; FREITAS, A. E. T. S.; SILVA, M. M. Composição da ictiofauna acompanhante da pesca de camarão em Tamandaré/PE e Pontal do Peba/AL. Bol. Téc. Cient. CEPENE, v. 6, n. 1, 1998.

SELLESLAGH, J.; AMARA, R.; LAFFARGUE, P.; LESOURD, S.; LEPAGE, M. \& GIRARDIN, M. 2009. Fish composition and assemblage structure in three Eastern English Channel Macrotidal estuaries: A comparison with other French estuaries. Estuar., Coast. Shelf Sci., v. 81, n. 2, p. 149-159, 2009.
SEPAQ (SECRETARIA DE ESTADO DA PESCA E AQUICULTURA). Estatística e Desembarque Pesqueiro do Estado do Pará - 2008. Belém: Governo do Estado do Pará, 2008. 28 p.

STOBUTZKI, I.; JONES, P.; MILLER, M. A comparison of fish bycatch communities between areas open and closed to prawn trawling in an Australia tropical fishery. ICES J. Mar. Sci., v. 60, n. 5, p. 951-966, 2003.

VÁSQUEZ, A. G.; ALONSO, J. C.; CARVAJAL, F.; MOREAU, J. N.; RENNO, J. F.; TELLO, S.; MONTREUIL, V.; DUPONCHELLE, F. Life-history characteristics of the large Amazonian migratory catfish Brachyplatystoma rousseauxii in the Iquitos region, Peru. J. Fish Biol., 75, p. 2527-2551, 2009.

VIANNA, M.; COSTA, F. E. S.; FERREIRA, C. N. Lengthweight relationship of fish caught as by-catch by shrimp fishery in the southeastern coast of Brazil. Bol. Inst. Pesca, v. 30, n. 1, p. 81 - 85, 2004.

YAÑEZ-ARANCIBIA, A.; SÀNCHEZ-GIL, P. Ecologia de los recursos demersales marinos. México: AGT Editor S.A., 1988. 228 p.

(Manuscript received 26 September 2012; revised 30 March 2013; accepted 30 April 2013) 